\title{
BMJ Open Impact of arthritis on the perceived need and use of mental healthcare among Canadians with mental disorders: nationally representative cross- sectional study
}

\author{
Alyssa Howren, ${ }^{1,2,3} \mathrm{~J}$ Antonio Aviña-Zubieta, ${ }^{2,4}$ Deborah Da Costa, ${ }^{5}$ \\ Joseph H Puyat, ${ }^{6}$ Hui Xie, ${ }^{2,7}$ Mary A De Vera (D) ${ }^{1,2,3}$
}

To cite: Howren A, AviñaZubieta JA, Da Costa D, et al. Impact of arthritis on the perceived need and use of mental healthcare among Canadians with mental disorders: nationally representative crosssectional study. BMJ Open 2020;10:e041371. doi:10.1136/ bmjopen-2020-041371

- Prepublication history for this paper is available online To view these files, please visit the journal online (http://dx.doi. org/10.1136/bmjopen-2020041371).

Received 09 June 2020 Revised 08 November 2020 Accepted 18 November 2020

Check for updates

(c) Author(s) (or their employer(s)) 2020. Re-use permitted under CC BY-NC. No commercial re-use. See rights and permissions. Published by BMJ.

For numbered affiliations see end of article.

Correspondence to

Dr Mary A De Vera;

mdevera@mail.ubc.ca

\section{ABSTRACT}

Objective To evaluate the association between having arthritis and the perceived need for mental healthcare and use of mental health support among individuals with mental disorders.

Design A cross-sectional analysis using data from Canadian Community Health Survey-Mental Health (2012).

Setting The survey was administered across Canada's 10 provinces using multistage cluster sampling.

Participants The study sample consisted of individuals reporting depression, anxiety or bipolar disorder.

Study variables and analysis The explanatory variable was self-reported doctor-diagnosed arthritis, and outcomes were perceived need for mental healthcare and use of mental health support. We computed overall and gender-stratified multivariable binomial logistic regression models adjusted for age, gender, race/ethnicity, income and geographical region.

Results Among 1774 individuals with a mental disorder in the study sample, 436 (20.4\%) reported having arthritis. Arthritis was associated with increased odds of having a perceived need for mental healthcare (adjusted OR (aOR) $1.71,95 \% \mathrm{Cl} 1.06$ to 2.77 ). In the gender-stratified models, this association was increased among men (aOR 2.69, $95 \% \mathrm{Cl} 1.32$ to 5.49 ) but not women (aOR $1.48,95 \% \mathrm{Cl}$ 0.78 to 2.82). Evaluation of the association between arthritis and use of mental health support resulted in an aOR of 1.50 (95\% Cl 0.89 to 2.51). Individuals with arthritis tended to use medications and professional services as opposed to non-professional support.

Conclusion Comorbid arthritis among individuals with a mental disorder was associated with an increased perceived need for mental healthcare, especially in men, underscoring the importance of understanding the role of masculinity in health seeking. Assessing the mental health of patients with arthritis continues to be essential for clinical care.

\section{INTRODUCTION}

Evidence from a Canadian population-based study suggests that having a physical chronic disease, such as diabetes or asthma, confers

\section{Strengths and limitations of this study}

- This cross-sectional study of how arthritis impacts the need and use of mental health support uses nationally representative data from an established Canadian population health survey.

- Our comprehensive assessment of mental health support included healthcare such as medications and professional services (eg, family doctor and psychiatrist), as well as non-professional forms of support (eg, friend and self-help group).

- Study limitations include self-reported arthritis status and the inability to ascertain temporality due to the cross-sectional study design.

a greater likelihood to receive psychological but not pharmacological treatment for comorbid mental disorder, namely, depression. ${ }^{1}$ Presumably, ongoing engagement with the healthcare system to manage a physical disease would help facilitate the diagnosis and treatment of mental disorders that otherwise might go unrecognised or untreated. ${ }^{2}$ However, the need for mental healthcare and the probability of receiving various forms of mental health support in individuals with arthritis are underappreciated, which is problematic, given the significant burden of comorbid mental disorders in arthritis. Indeed, recent systematic reviews estimate that the pooled prevalence of depression symptoms are $20 \%$ for individuals with osteoarthritis $^{3}$ and as high as 39\% for those with rheumatoid arthritis (RA). ${ }^{4}$ Further, Marrie et $a \check{l}$ in 2018 analysed Canadian populationbased administrative health data to show that individuals with RA have an increased risk for incident depression, anxiety and bipolar disorder. These mental disorders can exacerbate arthritis symptoms and lead to poor 
health outcomes. ${ }^{6-8}$ For example, depression and anxiety have been shown to delay joint remission, as measured with the Disease Activity Score, within the first year of RA diagnosis. ${ }^{9}$

Prior studies of mental healthcare use among patients with arthritis have primarily evaluated use of pharmacological treatment using descriptive approaches, including one study conducted in Greece reporting that only $13 \%$ and $12 \%$ of rheumatology patients with depression and anxiety, respectively, were prescribed antidepressants or anxiolytics. ${ }^{10}$ Conversely, a study in the USA found that $68 \%$ of those with self-reported arthritis and depression received antidepressants, and $19 \%$ received a combination of antidepressants and psychotherapy. ${ }^{11}$ Our understanding and efforts to improve mental health in the context of arthritis can be better informed by analytical studies that evaluate the independent effect of arthritis on the perceived need for mental healthcare, and also explore the use of mental health support beyond those that are pharmacological, to include psychological treatment, online therapy, self-help groups, and informal support from friends or family. As such, this study aimed to conduct a comprehensive population-based evaluation of the impact of arthritis on perceived need for mental healthcare and use of mental health support among individuals with mental disorders.

\section{MATERIALS AND METHODS}

\section{Study design and source population}

This population-based cross-sectional study used the public use microdata file from the nationally administered Canadian Community Health Survey-Mental Health (CCHS-MH) as the source population. In brief, the CCHS-MH was distributed in 2012 and aimed to assess the prevalence of psychological disorders, gaps in mental healthcare and use of mental health services. Survey sampling was allocated based on age, gender and geographical distribution to ensure representativeness at both the national and provincial levels. Individual participants 15 years and older across Canada's 10 provinces were then selected through multistage cluster sampling and were interviewed between January and December 2012. Approximately 3\% of the Canadian population is not represented as the CCHS-MH excludes individuals living on reserves, residing in one of the three territories, enrolled in the Canadian Armed Forces or currently institutionalised. In total, 25113 of 29088 eligible individuals participated in the survey, resulting in a person-level response rate of $86.3 \% .{ }^{12}$ Additional details on the design and methodology of the CCHS-MH are available through Statistics Canada. ${ }^{12}$ Data from the CCHS-MH were kept private and confidential as per the Statistics Act. ${ }^{13}$

\section{Study sample}

The analysis investigated the association between having arthritis and perceived need for mental healthcare, as well as use of mental health support among individuals with a mental disorder. As such, the study sample consisted of individuals who met criteria for major depressive episode, generalised anxiety disorder and/or bipolar disorder (bipolar I, bipolar II or hypomania) as defined by three derived variables based on screening questions in the CCHS-MH. The screening questions used to identify mental disorders in the CCHS-MH are derived from the WHO version of the Composite International Diagnostic Interview and arranged in the survey to resemble classifications from the Diagnostic and Safety Manual of Mental Disorders, Fourth Edition. All questions pertained to having depression, anxiety or bipolar disorder within the 12 months preceding the interview. Participants with invalid responses (ie, 'don't know', 'not stated' or 'refusal') to the derived variables for depression, anxiety and bipolar disorder were excluded. We further excluded individuals who did not have valid responses to the outcome variables, specifically perceived need for mental healthcare and the use of mental health support. Finally, participants were excluded if their responses to the explanatory variable, as well as confounding variables (eg, age, gender and income) were invalid (ie, don't know, not stated or refusal).

\section{Study variables}

Exposure

The main explanatory variable was self-reported doctordiagnosed arthritis status. Preceding the questions about chronic diseases such as arthritis, participants were prompted to consider chronic diseases such as those diagnosed by a health professional and lasting or expected to last a minimum of 6 months. The survey question specifically asked participants, 'Do you have arthritis, excluding fibromyalgia?' The arthritis variable was a binary categorical variable with a response option of yes or no.

\section{Outcomes}

The primary outcomes were (1) perceived need for mental healthcare in the past 12 months and (2) use of mental health support, specifically medications, professional services, or non-professional support for emotions, mental health or substance use within the 12 months prior to the CCHS-MH interview. The first outcome variable was constructed from a variable in the CCHS-MH that summarises a participants' perceived need for mental healthcare in the previous 12 months. This variable classifies participants into four categories (no perceived need, all perceived needs met, perceived needs partially met and perceived needs not met) based on their responses to having a perceived need for mental healthcare in terms of counselling, medications, information or other. We translated this variable into a binary indicator for whether individuals perceived to have a need for mental healthcare within the previous 12 months.

The second constructed outcome variable, referred to as 'use of mental health support', was a dichotomous indicator (yes or no) for use of one or more of the three aforementioned treatment or support modalities. 
Medication use referred to participants' use of any medications to help with emotions, mental health or substance use in the previous 12 months. Use of professional care included consultations provided by a psychiatrist, family doctor, psychologist, nurse, social worker/counsellor/ psychotherapist, as well as professional services by means of hospitalisation and online therapy. Support provided by non-professionals included assistance from a family member, friend, coworker, supervisor or boss, teacher or principal. Non-professional support also included self-help groups, services provided by an employer, telephone helplines or information gathered from the internet.

\section{Covariates}

We considered as potential confounders to the relationship between arthritis and outcomes of interest the following variables: age (10-year age groupings), gender (men and women), race/ethnicity (white and visible minority), household income (provincial level, by quintile) and geographical region (urban/rural). Geographical region was based on whether a participant resided in a 2011 census metropolitan area (population of $\geq 100000$, urban) or a non-census metropolitan area (rural).

\section{Statistical analysis}

Descriptive statistics were used to summarise demographics and perceived need and use of mental healthcare for individuals with and without arthritis. From the source population of all participants completing the CCHS-MH, we estimated the weighted prevalence of major depressive episode, generalised anxiety disorder and bipolar disorder among participants with and without arthritis. The primary analysis of the study sample applied multivariable binomial logistic regression to evaluate whether perceived need for care, as well as use of mental health support, differs by arthritis status, adjusted for the confounding effects of age, gender, race/ethnicity, household income and geographical region. We also stratified the primary analyses according to gender. We evaluated the relationship between arthritis status and use of specific mental health support (medications, professional services and non-professional support) using $\chi^{2}$ tests. The statistical analyses were weighted using CCHS-MH master weights to provide population-level estimates (eg, proportion (\%) and OR) and to account for sampling procedures. All of the analyses were completed using SAS University Edition (Release 3.8; SAS Institute Inc., Cary, NC, USA).

\section{Patient and public involvement}

Patients were not involved in the design, analysis or interpretation of results for this study. We plan to disseminate research findings to people living with arthritis through public forums and social media channels in collaboration with patient organisations, such as the Arthritis Patient Advisory Board of Arthritis Research Canada.
RESULTS

\section{Source population}

In the CCHS-MH source population consisting of 25113 participants in total, there were 5463 (16.7\%) individuals identified as having arthritis and 19628 (83.2\%) without arthritis (figure 1). Within the source population, depression was the most prevalent mental disorder in individuals with arthritis $(\mathrm{n}=326$, population weighted percentage $5.5 \%)$ followed by anxiety $(\mathrm{n}=231,4.3 \%)$ and bipolar disorder $(\mathrm{n}=88,1.6 \%)$. The prevalence of mental disorders was lower among individuals without arthritis, with weighted prevalence estimates of $4.5 \%(\mathrm{n}=991)$ for depression, $2.2 \% \quad(n=514)$ for anxiety and $1.5 \% \quad(n=295)$ for bipolar disorder.

\section{Study sample}

The study sample consisted of 1774 individuals with mental disorders, namely, depression, anxiety and/or bipolar disorder in the previous 12 months (figure 1). Descriptive characteristics for the study sample are reported in table 1. Among the study sample, 436 (20.4\%) individuals also reported having arthritis. Individuals with arthritis were older than those without arthritis, with $82.7 \%$ and $27.0 \%$ of participants over 45 years of age, respectively. There was a higher proportion of women among individuals with arthritis (67.3\% vs $58.9 \%$ no arthritis), and participants with arthritis tended to report lower household income. Finally, generalised anxiety disorder appeared to affect more participants with arthritis $(55.2 \%)$ compared with those without arthritis (35.2\%).

\section{Perceived need for mental healthcare}

Overall, 347 of $436(83.6 \%)$ participants with arthritis and 1028 of 1338 (75.7\%) participants without arthritis reported having a perceived need for mental healthcare. The unadjusted OR for the association between arthritis and having a perceived need for mental healthcare was 1.64 (95\% CI 1.08 to 2.48). In the multivariable model, adjusted for age, gender, race/ethnicity, income and geographical region, the positive association between arthritis and perceived need for mental healthcare persisted (adjusted OR (aOR) 1.71, 95\% CI 1.06 to 2.77) (table 2). A factor significantly associated with having a perceived need for mental healthcare was ethnicity/race. Analyses stratified by gender showed that among men, having arthritis was associated with increased odds for a perceived need for mental healthcare (aOR 2.69, 95\% CI 1.32 to 5.49$)$. The aOR among women with arthritis compared with women without arthritis was decreased and non-significant (aOR 1.48, 95\% CI 0.78 to 2.82).

Among those participants indicating a perceived need for care in the previous 12 months, participants without arthritis $(12.9 \%)$ were more likely to report that their perceived need for care was 'not met' as compared with those with arthritis (7.0\%). Despite this disparity, the majority of participants both with $(96.0 \%)$ and without $(93.5 \%)$ arthritis who had a perceived need for mental healthcare also reported that they used mental health 

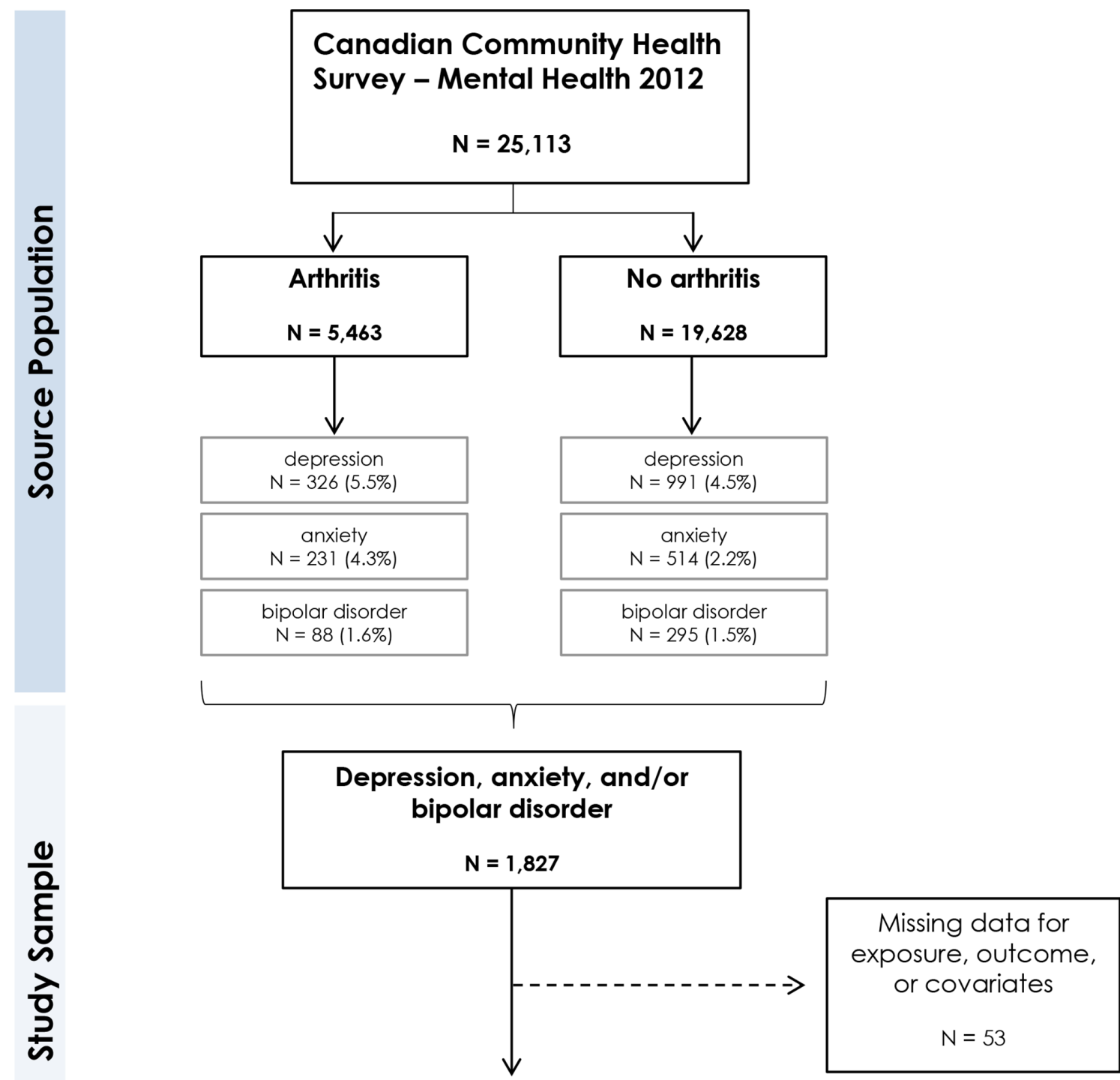

Final study sample

$N=1,774$

Figure 1 Flow diagram for creation of study sample from the Canadian Community Health Survey-Mental Health ${ }^{12}$ and indication of samples used for analyses. All percentages reported are weighted using probability weights from Statistics Canada.

support (ie, medication, professional service and/or nonprofessional support) in the previous 12 months. Stratification by gender in the arthritis sample revealed that a slightly higher proportion of women (97.9\%) compared with men $(92.5 \%)$ having a perceived need for mental healthcare reported using mental health support.

\section{Use of mental health support}

The majority of participants with mental disorders used at least one type of mental health support (ie, medication, professional service and/or non-professional support) and the prevalence of use was similar among those with arthritis $(82.8 \%)$ and without arthritis $(79.3 \%)$. In the unadjusted analysis, the odds of using mental health support among individuals with arthritis compared with those without arthritis was 1.26 (95\% CI 0.81 to 1.97$)$. In the adjusted analysis that accounted for the confounding effects of age, gender, race/ethnicity, income and geographical region, the aOR for the association between arthritis and mental health support was 1.50 (95\% CI 0.89 to 2.51). Additional details for the unadjusted and adjusted analyses are shown in table 2. Assessment of the model covariates suggests that women are more likely to use mental health support compared with men. In the gender-stratified analysis, aORs for the association between arthritis and mental health support remained similar among women (aOR $1.78,95 \%$ CI 0.90 to 3.52 ) and men (aOR 1.52, 95\% CI 0.64 to 3.66).

The type of mental health support predominately used by individuals with arthritis were professional services (66.8\% arthritis, $58.1 \%$ no arthritis; $\mathrm{p}$ value $=0.0357$ ) 
Table 1 Descriptive characteristics of a Canadian Community Health Survey -Mental Health (2012) study sample with mental disorders $(\mathrm{N}=1774)$

\begin{tabular}{|c|c|c|}
\hline & $\begin{array}{l}\text { Arthritis } \\
(\mathrm{N}=436) \mathrm{N}(\%)\end{array}$ & $\begin{array}{l}\text { No arthritis } \\
(\mathrm{N}=1338) \mathrm{N}(\%)\end{array}$ \\
\hline \multicolumn{3}{|l|}{ Gender } \\
\hline Men & $127(32.7)$ & $509(41.1)$ \\
\hline Women & $309(67.3)$ & $829(58.9)$ \\
\hline \multicolumn{3}{|l|}{ Age (years) } \\
\hline $15-24$ & $6(0.9)$ & $352(27.3)$ \\
\hline $25-34$ & $18(5.3)$ & $285(23.1)$ \\
\hline $35-44$ & $42(11.1)$ & $263(22.5)$ \\
\hline $45-54$ & $99(24.6)$ & $236(16.6)$ \\
\hline $55-64$ & $162(39.1)$ & $148(7.0)$ \\
\hline $65-74$ & $73(12.1)$ & $36(2.6)$ \\
\hline$\geq 75$ & $36(6.9)$ & $18(0.8)$ \\
\hline \multicolumn{3}{|l|}{ Race/ethnicity } \\
\hline Visible minority & $58(17.1)$ & $237(23.6)$ \\
\hline White & 378 (82.9) & $1101(76.4)$ \\
\hline \multicolumn{3}{|l|}{ Household income } \\
\hline Quintile 1 (lowest) & $212(41.1)$ & $412(28.2)$ \\
\hline Quintile 2 & 98 (24.6) & $273(22.6)$ \\
\hline Quintile 3 & $61(15.6)$ & $250(20.7)$ \\
\hline Quintile 4 & 45 (12.4) & $231(15.9)$ \\
\hline Quintile 5 (highest) & $20(6.3)$ & $172(12.7)$ \\
\hline \multicolumn{3}{|l|}{ Geographical region } \\
\hline Urban & $243(71.8)$ & $849(72.6)$ \\
\hline Rural & $193(28.2)$ & $489(27.4)$ \\
\hline \multicolumn{3}{|l|}{ Mental disorder ${ }^{\star}$} \\
\hline Major depressive episode & $316(69.1)$ & $961(72.8)$ \\
\hline $\begin{array}{l}\text { Generalised anxiety } \\
\text { disorder }\end{array}$ & $222(55.2)$ & $502(35.2)$ \\
\hline Bipolar disorder & $85(21.0)$ & $290(24.1)$ \\
\hline
\end{tabular}

All percentages reported are weighted using probability weights from Statistics Canada.

*Participants can have more than one mental disorder.

and medication $(62.3 \%$ arthritis, $45.1 \%$ no arthritis; $p$ value $=0.0001$ ). Evaluation of the specific forms of professional services used by participants indicates that a higher proportion of individuals with arthritis seek care from their family doctor $(52.6 \%$ arthritis, $40.4 \%$ no arthritis) and/or psychiatrist (24.0\% arthritis, $17.7 \%$ no arthritis). In contrast, non-professional support was more frequently used by individuals without arthritis (67.3\%) compared with those with arthritis $(51.6 \%)$ (p value $=0.0002$ ). This disparity was predominately seen as a greater tendency for individuals without arthritis to talk with family members (29.9\% arthritis, $43.8 \%$ no arthritis) and friends (34.2\% arthritis, $43.9 \%$ no arthritis) about their mental health, and to use the internet for information, help or support (23.5\% arthritis, $33.3 \%$ no arthritis).
A full description of specific professional services and non-professional support is provided in figures 2 and 3 , respectively. Among those with arthritis, stratification by gender shows a similar usage pattern for each type of mental health support (medication: $63.1 \%$ men, $62.0 \%$ women; professional services: $67.9 \%$ men, $66.3 \%$ women; non-professional support: $52.5 \%$ men, $51.2 \%$ women).

\section{DISCUSSION}

The aim of this study was to use data from an established nationally representative survey in Canada to evaluate the relationship between arthritis and the perceived need for mental healthcare, as well as use of mental health support among individuals with mental disorders. Findings suggest that among individuals with a mental disorder, arthritis is associated with $71 \%$ higher odds of having a perceived need for mental healthcare, and that this association only remained significant among men when stratifying the analysis by gender. Results also suggest similar odds for individuals with arthritis to use mental health support as compared with those without arthritis. An exploration into the three different types of mental health support suggests that pharmacological treatment and professional services may be the more sought-after support for individuals with arthritis, with fewer seeking non-professional forms of support. These findings may reflect the increasing recognition of the importance of mental health in arthritis and emphasise the necessity of engaging in discussions around mental health needs and support with patients and to ensure strategies employed are informed by the role of gender. ${ }^{4115}$

Although there is a demonstrated burden of mental disorders among the arthritis population, there is very limited research on how arthritis independently affects an individual's perceived need and use of mental healthcare. Results from our study describe a higher perceived need for mental healthcare among individuals with arthritis, and importantly after stratification by gender, showed that men with arthritis have 2.69 times higher odds than men without arthritis in sensing a need for care, a much stronger association than that observed among women. Interestingly, the influence of gender did not persist when the outcome of interest was use of mental health support. It is possible that while men diagnosed with arthritis and a mental disorder perceive a need for mental healthcare, the conformity to traditional masculine norms and the presence of stigma negatively impact health seeking and therefore use of mental health support. ${ }^{16}$ Qualitative findings from Seidler et al $\mathrm{s}^{16} 2016$ systematic review reported that men often misinterpreted the psychological symptoms of depression, such as fatigue and irritability, as signs of a physical ailment. Indeed, chronic conditions are known risk factors for mental disorders such as depression ${ }^{17}$ and anxiety ${ }^{18}$; however, the symptom overlap (eg, pain and fatigue) between these physical and mental illnesses often leads to mental disorders being undetected. Overall, the increased perceived need for mental healthcare among 
Table 2 Unadjusted and adjusted logistic regression models evaluating the association between arthritis and (1) perceived need for mental healthcare and (2) use of mental health support among individuals with mental disorders, Canadian Community Health Survey-Mental Health sample (2012)

Perceived need for mental healthcare* (yes vs no) Use of mental health support* (yes vs no) OR $(95 \% \mathrm{Cl})$ OR $(95 \% \mathrm{Cl})$

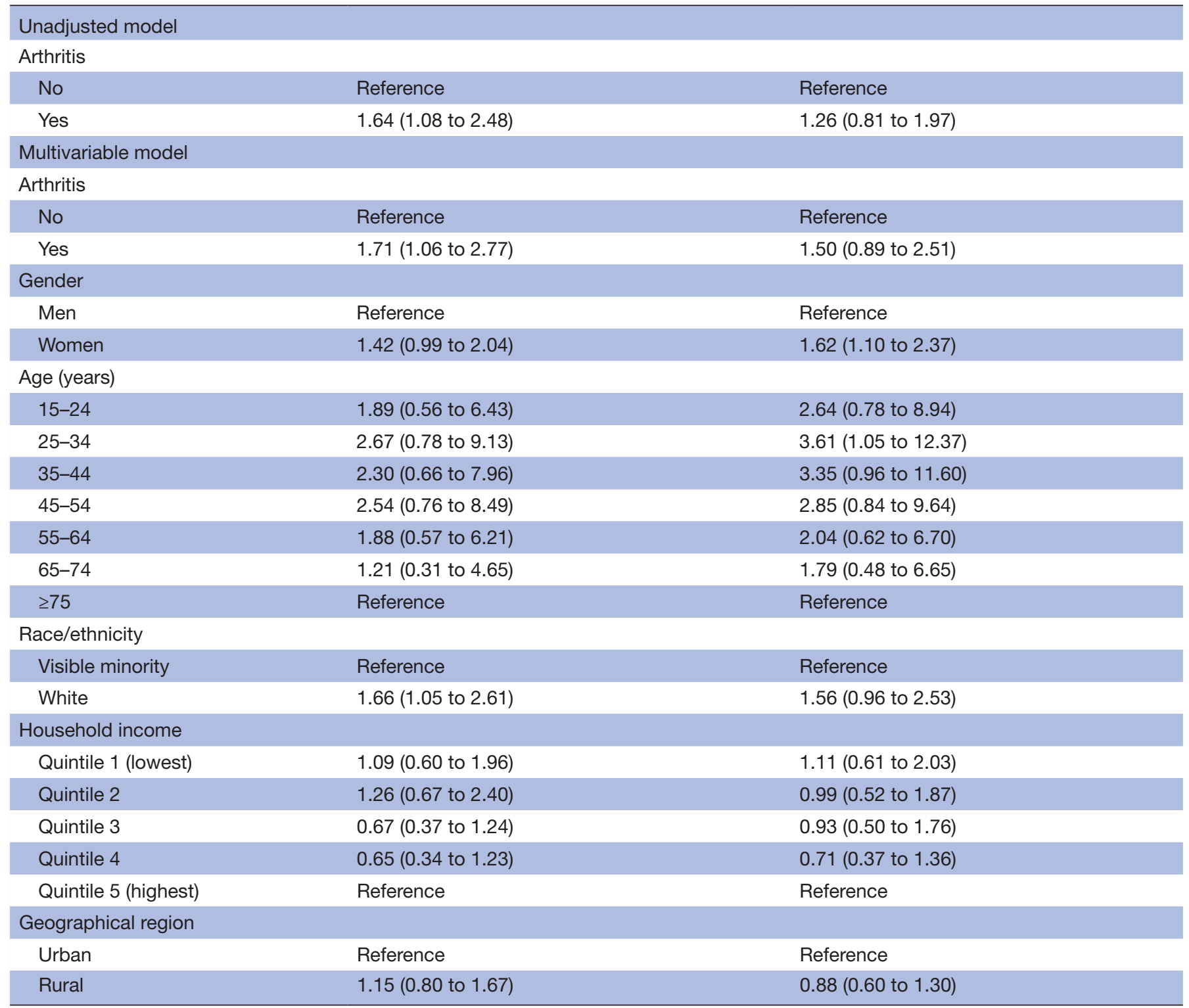

Estimates are probability weighted using weights from Statistics Canada.

*Adjusted for gender, age, race/ethnicity, income and geographical region.

individuals with arthritis emphasises the importance of monitoring the mental health of patients with arthritis and being mindful of gender-specific symptom presentation and health-seeking behaviours, which may require custom support strategies. Future research to characterise mental health needs for this patient population includes understanding how various biopsychosocial factors, such as pain, functional capacity and illness adjustment, influence the perceived need for mental healthcare among individuals with arthritis.

The lack of an association between arthritis and mental health support in our study suggests similar odds for use of mental health support among individuals with arthritis compared with those without arthritis. These findings are supported by a cross-sectional study conducted by Wittayanukorn et $a l^{19}$ in 2014 that identified predictors of receiving depression treatment among 1177 respondents to the US National Health and Nutrition Examination Survey from 2005 to 2010. In this study, authors noted that having arthritis was a significant predictor of using pharmacological or professional services among those with depression (OR 1.5, 95\% CI 1.1 to 2.1), and although study purposes differed, our study resulted in a similar estimate (aOR $1.50,95 \%$ CI 0.89 to 2.51$).{ }^{19} \mathrm{~A}$ 


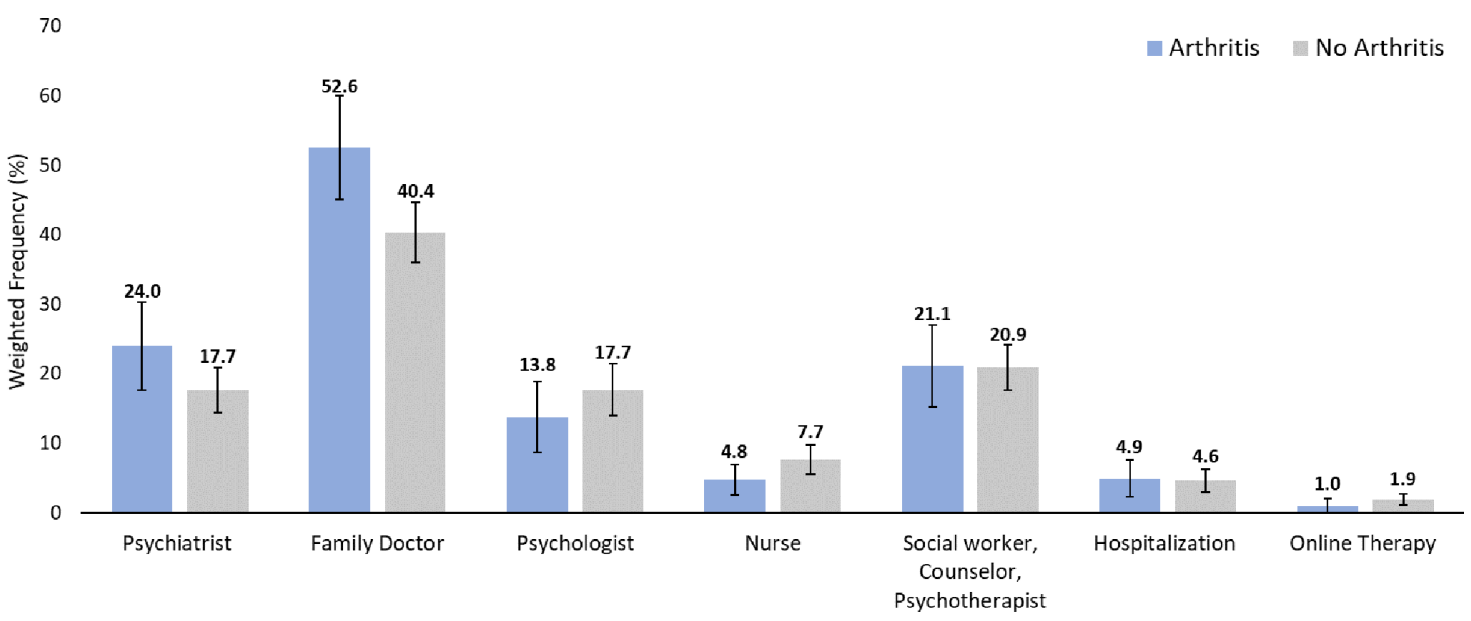

Figure 2 Population weighted frequencies (95\% Cls) of professional consultations or services used in the previous 12 months according to arthritis status. All percentages reported are weighted using probability weights from Statistics Canada.

finding unique to our study is the predominance of individuals with arthritis seeking clinical forms of mental healthcare, such as medication or professional services, and fewer turning to non-professional forms of care and support, such as family, friends or support groups. While a number of previous studies in arthritis have investigated the use of medications and professional services, ${ }^{10} 111920$ the decreased use of non-professional support is a novel contribution to understanding how patients with arthritis seek care for their mental health. It is plausible that individuals with arthritis are more inclined to access medication or professional services because of their familiarity with the healthcare system, where clinical guidelines to manage depression, for example, recommend antidepressants and psychotherapy as first-line treatment. ${ }^{21}{ }^{22}$ Severity may also be a contributing factor, as those with arthritis in our study sample were older and may have had a mental health condition for a longer period, thus requiring professional and/or pharmacological treatment, and may have already exhausted the use of non-professional forms of support. In addition, the social isolation and loneliness secondary to arthritis symptoms ${ }^{23}$ may translate to individuals avoiding discussions about mental health symptoms with relatives and peers. It is imperative that future interventions for mental disorders in individuals with arthritis consider the complexities of managing arthritis and address the social aspects of treatment, ${ }^{24}$ especially given that interventions trialled to date are primarily clinical. ${ }^{2526}$ Further, clinical practice guidelines for arthritis should consider including guidance on mental health management that is in alignment with existing mental health guidelines. Depression guidelines from the Canadian Network for Mood and Anxiety Treatments, for example, recommend screening for depression among those with chronic medical conditions and chronic pain when resources and services are available. ${ }^{17}$

Strengths of this study include evaluation of the independent association between arthritis and the perceived need for mental healthcare, as well as use of mental health support using data from a nationally representative

60

Arthritis No Arthritis

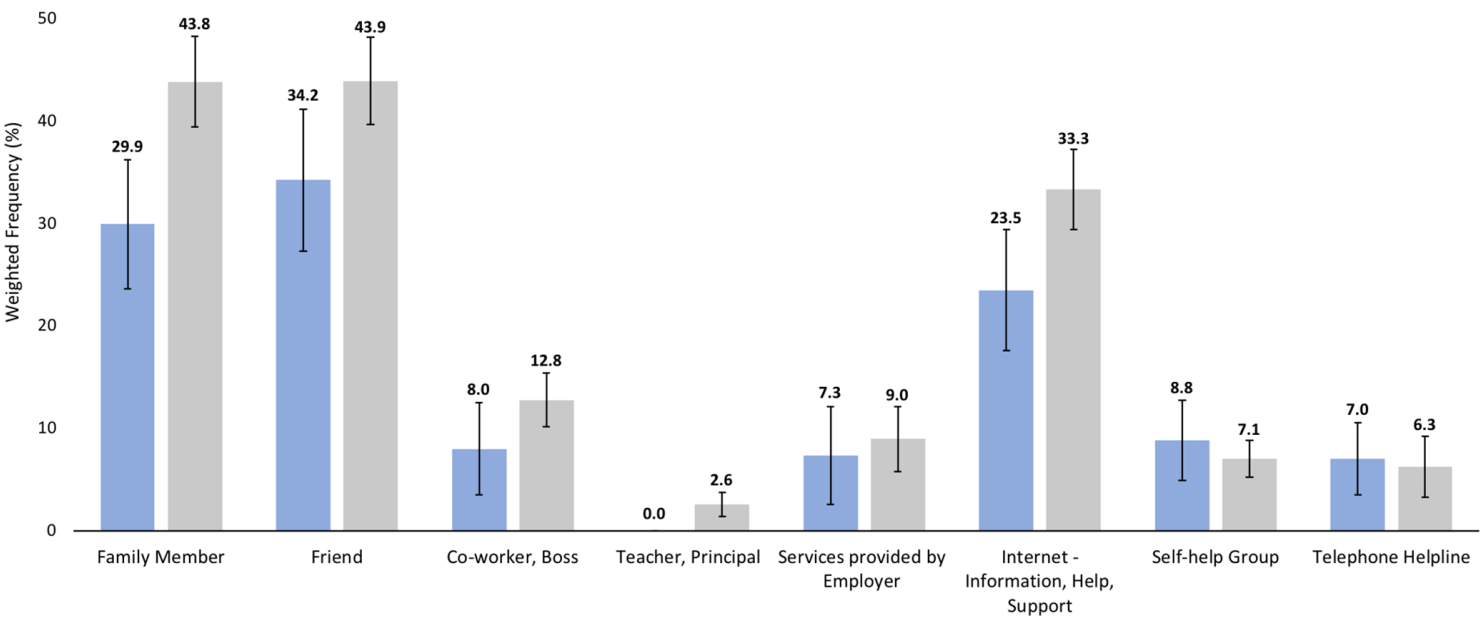

Figure 3 Population weighted frequencies (95\% Cls) of non-professional forms of support used in the previous 12 months according to arthritis status. All percentages reported are weighted using probability weights from Statistics Canada. 
sample. This is also the first study to comprehensively assess mental health support by including non-professional support seeking along with medications and professional services. Assessment of covariates for the overall model shows that women had higher odds of receiving mental health support compared with men. This finding is consistent with gender differences reported in previous studies, where women are more likely than men to use mental health services and be prescribed medications, ${ }^{27} 28$ and therefore adds validity to the model and the main findings. There are also limitations to consider when interpreting the results of this study. First, the identification of individuals with arthritis is based on self-reported data as opposed to physician-diagnosed data. However, the CCHS-MH survey asks participants to report only chronic diseases confirmed by a physician, and self-reported arthritis has been shown to be reasonably valid and reliable. ${ }^{29}{ }^{30}$ Moreover, our study is restricted to the variables included in the CCHS-MH survey, which does not include information on arthritis type and disease severity. There is also a possibility of systematic error due to respondent bias. In particular, individuals with arthritis may be better able to recall their treatment history, given their ongoing clinical management and awareness of personal health status. Lastly, the cross-sectional study design precludes the ability to infer temporality between exposure and outcome variables.

The current study suggests that individuals with arthritis have a greater perceived need for mental healthcare and similar odds of receiving support for their mental disorders in comparison to the general population. The implications of these findings are that while those with arthritis may have a greater perceived need for mental healthcare, mental health support in the form of medication and professional consults or services are being accessed, perhaps as a result of established connections with the healthcare system and/or having had a mental disorder for a longer duration. The potential role of traditional masculine norms in symptom recognition and healthcare seeking for mental disorders highlight additional opportunities for improving mental health in individuals with arthritis. To obtain a clearer understanding of these associations, it is important to explore non-professional forms of support as well as to conduct longitudinal analyses as this will enable us to understand temporal trends and to assess causality.

\section{Author affiliations}

${ }^{1}$ Faculty of Pharmaceutical Sciences, University of British Columbia, Vancouver, British Columbia, Canada

${ }^{2}$ Arthritis Research Canada, Richmond, British Columbia, Canada

${ }^{3}$ Collaboration for Outcomes Research and Evaluation, Vancouver, British Columbia, Canada

${ }^{4}$ Faculty of Medicine, Department of Medicine, Division of Rheumatology, University of British Columbia, Vancouver, British Columbia, Canada

${ }^{5}$ Faculty of Medicine, Department of Medicine, McGill University, Montreal, Quebec, Canada

${ }^{6}$ Faculty of Medicine, School of Population and Public Health, University of British Columbia, Vancouver, British Columbia, Canada
${ }^{7}$ Faculty of Health Sciences, Simon Fraser University, Burnaby, British Columbia, Canada

Twitter Mary A De Vera @maryadevera

Contributors AH and MDV contributed to the study design, as well as acquisition, analysis and interpretation of data. JAA-Z, DDC, JHP and HX contributed to study design, data analysis and interpretation. AH drafted the manuscript and all study authors (AH, JAA-Z, DDC, JHP, HX and MDV) revised and approved the final version for publication.

Funding This work was supported by a Strategic Operating Grant from The Arthritis Society (\#SOG-18-0192).Dr. De Vera holds a Canada Research Chair in Medication Adherence, Utilization, and Outcomes and is The Arthritis Society Network Scholar and Michael Smith Foundation for Health Research Scholar.Mrs. Howren is a recipient of a Canadian Institutes of Health Research Frederick Banting and Charles Best Canada Graduate Scholarship Doctoral Award and a Wayne Riggs Interdisciplinary Doctoral Scholarship in Pharmaceutical Sciences (University of British Columbia).Dr. Aviña-Zubieta is the British Columbia Lupus Society Research Scholar and the Walter \& Marilyn Booth Research Scholar.Dr. Xie is Milan and Maureen Illich/Merck Frosst Chair in Biostatistics for Arthritis and Musculoskeletal Diseases.

Competing interests None declared.

Patient and public involvement Patients and/or the public were not involved in the design, conduct, reporting or dissemination plans of this research.

Patient consent for publication Not required.

Ethics approval Ethical approval to use the publicly available Canadian Community Health Survey — Mental Health data was covered by University of British Columbia's Policy (\#LR9) on Research Involving Human Participants (https:// universitycounsel-2015.sites.olt.ubc.ca/files/2019/08/Human-Research-Policy_ LR9.pdf).

Provenance and peer review Not commissioned; externally peer reviewed.

Data availability statement Data are available in a public, open access repository. The public use microdata file can be accessed through Statistics Canada (https:// www150.statcan.gc.ca/n1/en/catalogue/82M0021X).

Open access This is an open access article distributed in accordance with the Creative Commons Attribution Non Commercial (CC BY-NC 4.0) license, which permits others to distribute, remix, adapt, build upon this work non-commercially, and license their derivative works on different terms, provided the original work is properly cited, appropriate credit is given, any changes made indicated, and the use is non-commercial. See: http://creativecommons.org/licenses/by-nc/4.0/.

ORCID iD

Mary A De Vera http://orcid.org/0000-0002-2205-2683

\section{REFERENCES}

1 Puyat JH, Kazanjian A, Wong $\mathrm{H}$, et al. Comorbid chronic general health conditions and depression care: a population-based analysis. Psychiatr Serv 2017;68:907-15.

2 Fikretoglu D, Liu A. Perceived barriers to mental health treatment among individuals with a past-year disorder onset: findings from a Canadian population health survey. Soc Psychiatry Psychiatr Epidemiol 2015;50:739-46.

3 Stubbs B, Aluko Y, Myint PK, et al. Prevalence of depressive symptoms and anxiety in osteoarthritis: a systematic review and meta-analysis. Age Ageing 2016;45:228-35.

4 Matcham F, Rayner L, Steer S, et al. The prevalence of depression in rheumatoid arthritis: a systematic review and meta-analysis. Rheumatology 2013;52:2136-48.

5 Marrie RA, Walld R, Bolton JM, et al. Increased incidence of psychiatric disorders in immune-mediated inflammatory disease. $J$ Psychosom Res 2017;101:17-23.

6 Marengo MF, Waimann CA, de Achaval S, et al. Measuring therapeutic adherence in systemic lupus erythematosus with electronic monitoring. Lupus 2012;21:1158-65.

7 Ang DC, Choi H, Kroenke K, et al. Comorbid depression is an independent risk factor for mortality in patients with rheumatoid arthritis. J Rheumatol 2005;32:1013-9.

8 Hyphantis T, Kotsis K, Tsifetaki N, et al. The relationship between depressive symptoms, illness perceptions and quality of life in 
ankylosing spondylitis in comparison to rheumatoid arthritis. Clin Rheumatol 2013;32:635-44.

9 Boer AC, Huizinga TWJ, van der Helm-van Mil AHM. Depression and anxiety associate with less remission after 1 year in rheumatoid arthritis. Ann Rheum Dis 2019;78:e1.

10 Anyfanti P, Gavriilaki E, Pyrpasopoulou A, et al. Depression, anxiety, and quality of life in a large cohort of patients with rheumatic diseases: common, yet undertreated. Clin Rheumatol 2016;35:733-9.

11 Agarwal P, Pan X, Sambamoorthi U. Depression treatment patterns among individuals with osteoarthritis: a cross sectional study. BMC Psychiatry 2013;13:121.

12 Statistics Canada. Canadian community health survey - mental health (CCHS) [cited October 13, 2018], 2012. Available: http:// www23.statcan.gc.ca/imdb/p2SV.pl?Function=getSurvey\&SDDS= 5015

13 Government of Canada. Statistics act (r.S.C., 1985, C. S-19) [cited October 13, 2018]. Available: https://laws-lois.justice.gc.ca/PDF/S19.pdf

14 Margaretten M, Julian L, Katz P, et al. Depression in patients with rheumatoid arthritis: description, causes and mechanisms. Int J Clin Rheumtol 2011;6:617-23.

15 Zhang L, Fu T, Yin R, et al. Prevalence of depression and anxiety in systemic lupus erythematosus: a systematic review and metaanalysis. BMC Psychiatry 2017;17:70.

16 Seidler ZE, Dawes AJ, Rice SM, et al. The role of masculinity in men's help-seeking for depression: a systematic review. Clin Psychol Rev 2016;49:106-18.

17 Lam RW, McIntosh D, Wang J, et al. Canadian network for mood and anxiety treatments (CANMAT) 2016 clinical guidelines for the management of adults with major depressive disorder: section 1. disease burden and principles of care. Can J Psychiatry 2016;61:510-23.

18 Katzman MA, Bleau P, Blier P, et al. Canadian clinical practice guidelines for the management of anxiety, posttraumatic stress and obsessive-compulsive disorders. BMC Psychiatry 2014;14 Suppl $1:$ S1.
19 Wittayanukorn S, Qian J, Hansen RA. Prevalence of depressive symptoms and predictors of treatment among U.S. adults from 2005 to 2010. Gen Hosp Psychiatry 2014;36:330-6.

20 Jyrkkä J, Kautiainen $\mathrm{H}$, Koponen $\mathrm{H}$, et al. Antidepressant use among persons with recent-onset rheumatoid arthritis: a nationwide registerbased study in Finland. Scand J Rheumatol 2014;43:364-70.

21 Kennedy SH, Lam RW, Mclntyre RS, et al. Canadian network for mood and anxiety treatments (CANMAT) 2016 clinical guidelines for the management of adults with major depressive disorder: section 3. pharmacological treatments. Can J Psychiatry 2016;61:540-60.

22 Parikh SV, Quilty LC, Ravitz P, et al. Canadian network for mood and anxiety treatments (CANMAT) 2016 clinical guidelines for the management of adults with major depressive disorder: section 2 . psychological treatments. Can J Psychiatry 2016;61:524-39.

23 Arthritis Action. Arthritis: overcoming the challenges [cited December 11, 2018], 2016. Available: https://www.arthritisaction.org.uk/wpcontent/uploads/2016/08/AA-report-WEB.pdf

24 Johnson S. Social interventions in mental health: a call to action. Soc Psychiatry Psychiatr Epidemiol 2017;52:245-7.

25 Fiest KM, Hitchon CA, Bernstein CN, et al. Systematic review and meta-analysis of interventions for depression and anxiety in persons with rheumatoid arthritis. J Clin Rheumatol 2017;23:425-34.

26 Zhang J, Wei W, Wang CM. Effects of psychological interventions for patients with systemic lupus erythematosus: a systematic review and meta-analysis. Lupus 2012;21:1077-87.

27 Kessler RC, Demler O, Frank RG, et al. Prevalence and treatment of mental disorders, 1990 to 2003. N Engl J Med 2005;352:2515-23.

28 Smith KLW, Matheson Fl, Moineddin R, et al. Gender differences in mental health service utilization among respondents reporting depression in a national health survey. Health 2013;05:1561-71.

29 Bombard JM, Powell KE, Martin LM, et al. Validity and reliability of self-reported arthritis: Georgia senior centers, 2000-2001. Am J Prev Med 2005:28:251-8.

30 Picavet HSJ, Hazes JMW. Prevalence of self reported musculoskeletal diseases is high. Ann Rheum Dis 2003;62:644-50. 